THE ASTROPHYSICAL JOURNAL, 302:675-679, 1986 March 15

c 1986. The American Astronomical Society. All rights reserved. Printed in U.S.A.

\title{
TWO SUPERGIANTS IN THE LARGE MAGELLANIC CLOUD WITH THICK DUST SHELLS
}

\author{
J. H. ELIAS ${ }^{1}$ \\ California Institute of Technology \\ JAY A. Frogel \\ Cerro Tololo Inter-American Observatory, National Optical Astronomy Observatories \\ AND \\ P. B. W. SCHWERING \\ Sterrewacht, Leiden \\ Received 1985 July 11; accepted 1985 September 17
}

\begin{abstract}
Ground-based observations from 0.6 to $20 \mu \mathrm{m}$ have identified two luminous, evolved stars surrounded by thick dust shells among the Magellanic Cloud sources detected by the Infrared Astronomical Satellite. Their energy distributions resemble those of typical Galactic OH/IR stars, but they have bolometric magnitudes brighter than -9 and the small-amplitude variability of supergiants. One star, IRAS $04553-6825$, has a spectral type of M7.5 and a dust shell which absorbs and reradiates roughly $75 \%$ of the star's luminosity; its radial velocity confirms its LMC membership. The second star, IRAS 05346-6949, has an even thicker dust shell, and the central star is not observable.
\end{abstract}

Subject headings: galaxies: Magellanic Clouds - stars: circumstellar shells — stars: supergiants

\section{INTRODUCTION}

The Infrared Astronomical Satellite (IRAS: Neugebauer et al. 1984), during the course of its mission, surveyed most of the sky at wavelengths of $12,25,60$, and $100 \mu \mathrm{m}$. Several hundred sources were detected at 12 and $25 \mu \mathrm{m}$ in the Large and Small Magellanic Clouds (LMC and SMC), mainly in the LMC. For many of these, there is no unambiguous identification with a previously known object; thus a program of ground-based observations of LMC and SMC IRAS sources was begun.

The ground-based work shows that the Magellanic Cloud $I R A S$ sources fall into three categories: foreground stars, highly evolved stars, and newly formed stars. In the Galaxy, highly evolved stars can develop dust shells of significant optical depth. Such objects were first detected by the TwoMicron Sky Survey (Neugebauer and Leighton 1969); more extreme examples were found by the AFGL Sky Survey (Price and Walker 1976; Price 1977). Most are stars of a few solar masses on the asymptotic giant branch (AGB), but a few appear to be supergiants, with higher masses (see the review by Herman and Habing 1985). In the SMC, AGB stars of similar luminosity are also present, though with far less dust surrounding them (Elias, Frogel, and Humphreys 1980, 1985; Wood, Bessell, and Fox 1983). There are no known SMC supergiants with thick dust shells. The similar absence of stars with extensive dust shells from the LMC (Wood, Bessell, and Fox 1983; Elias, Frogel, and Humphreys 1985) has been explained either as a genuine deficiency, or as due to the limitation of survey techniques used to find them.

This paper reports on two objects in the LMC discovered during the first season of ground-based observations; we will

\footnotetext{
${ }^{1}$ Visiting Astronomer, Cerro Tololo Inter-American Observatory, National Optical Astronomy Observatories, which are operated by the Association of Universities for Research in Astronomy under contract with the National Science Foundation.
}

describe the remaining sources and the details of the groundbased follow-up in a later paper.

\section{OBSERVATIONS AND RESULTS}

The positions of IRAS sources in the LMC with a flux density greater than $2 \mathrm{Jy}$ at $12 \mu \mathrm{m}$ were searched at $10 \mu \mathrm{m}$ on the CTIO $1.5 \mathrm{~m}$ telescope, using the $\mathrm{f} / 30$ wobbling secondary and germanium bolometer (Dewar "D-2") with a 12".7 beam. Strong sources that were found were also measured at $20 \mu \mathrm{m}$, and from 1.2 to $4.8 \mu \mathrm{m}$ with the InSb detector (Dewar "D-3"). Source positions were derived from the encoders of the $1.5 \mathrm{~m}$ telescope; measurements of apparent positions of nearby SAO stars indicate that the errors in these coordinates should be no worse than $3^{\prime \prime}$. Further observations of the brighter sources were made on the CTIO $4 \mathrm{~m}$ telescope and $\mathrm{f} / 30$ wobbling secondary, using a 5 " beam. Measurements were made through six narrow-band $10 \mu \mathrm{m}$ ("silicate") filters as well as through the broad-band $1.2-20 \mu \mathrm{m}$ filters used on the $1.5 \mathrm{~m}$ telescope.

For some of the sources that had apparent visual counterparts, moderate-resolution spectra were also taken covering 6200-9200 A. These were done on the $4 \mathrm{~m}$ telescope and RC spectrograph using the air Schmidt camera and GEC CCD detector. The resolution was $\sim 6 \AA$.

Two of the IRAS sources observed at CTIO appear spatially unresolved, with energy distributions characteristic of late-type stars surrounded by extensive circumstellar dust shells. These two are among the most luminous late-type stars in the LMC and are comparable to the most luminous Galactic stars of similar type. Positions and infrared photometry for these two sources are given in Table 1. A summary of the relevant IRAS data for the two objects is given in Table 2. It should be noted that the dominant source of uncertainty at 60 and $100 \mu \mathrm{m}$ is confusion with other, nearby sources. Energy distributions for the two objects are shown in Figures 1 and 2, together with distributions of some comparison objects. IRAS 04553-6825 
TABLE 1

Ground-Based Positions and Magnitudes of IRAS ObJects

\begin{tabular}{|c|c|c|}
\hline Position/Magnitude & $I R A S 04553-6825$ & $I R A S 05346-6949$ \\
\hline \multicolumn{3}{|l|}{ Positions: ${ }^{\mathrm{a}}$} \\
\hline$\alpha(1950) \ldots$ & $4^{\mathrm{h}} 55^{\mathrm{m}} 18^{\mathrm{s}} .0$ & $5^{\mathrm{h}} 34^{\mathrm{m}} 40^{\mathrm{s}} .5$ \\
\hline$\delta(1950) \ldots \ldots \ldots \ldots \ldots \ldots \ldots$ & $-68^{\circ} 25^{\prime} 16^{\prime \prime}$ & $-69^{\circ} 49^{\prime} 20^{\prime \prime}$ \\
\hline \multicolumn{3}{|l|}{ Broad-Band Magnitudes:b } \\
\hline$J \ldots \ldots \ldots \ldots \ldots \ldots \ldots$ & $9.52 \pm 0.04$ & $\ldots$ \\
\hline$H \ldots$ & $8.07 \pm 0.04$ & $\ldots$ \\
\hline .................. & $6.88 \pm 0.04$ & $16.0 \pm 0.2^{\mathrm{c}}$ \\
\hline$\ldots \ldots \ldots \ldots \ldots \ldots \ldots$ & $5.04 \pm 0.03$ & $9.86 \pm 0.07$ \\
\hline$[4.7] \ldots$ & $3.82 \pm 0.04$ & $6.51 \pm 0.08$ \\
\hline$[10] \ldots$ & $1.74 \pm 0.10$ & $2.00 \pm 0.07$ \\
\hline$[20] \ldots \ldots \ldots \ldots \ldots \ldots \ldots \ldots \ldots$ & $+0.1 \pm 0.4^{\mathrm{d}}$ & $-0.5 \pm 0.4^{\mathrm{d}}$ \\
\hline \multicolumn{3}{|l|}{ Narrow-Band Magnitudes: ${ }^{b}$} \\
\hline$[7.8] \ldots \ldots \ldots \ldots \ldots \ldots \ldots$ & $2.50 \pm 0.15$ & $2.83 \pm 0.15$ \\
\hline$[8.6] \ldots \ldots \ldots \ldots \ldots \ldots \ldots \ldots$ & $2.12 \pm 0.09$ & $2.57 \pm 0.09$ \\
\hline$[9.6] \ldots \ldots \ldots \ldots \ldots \ldots \ldots$ & $2.17 \pm 0.06$ & $2.87 \pm 0.06$ \\
\hline$[10.4] \ldots \ldots \ldots \ldots \ldots \ldots \ldots \ldots$ & $1.74 \pm 0.04$ & $2.27 \pm 0.04$ \\
\hline$[11.4] \ldots \ldots \ldots \ldots \ldots \ldots \ldots$ & $1.46 \pm 0.12$ & $1.68 \pm 0.12$ \\
\hline$[12.4] \ldots \ldots \ldots \ldots \ldots \ldots \ldots \ldots$ & $1.13 \pm 0.10$ & $0.91 \pm 0.10$ \\
\hline $\mathrm{H}_{2} \mathrm{O} \ldots \ldots \ldots \ldots \ldots \ldots$ & $+0.84 \pm 0.03$ & $\ldots$ \\
\hline CO $\ldots \ldots \ldots \ldots \ldots \ldots \ldots$ & $-0.09 \pm 0.01$ & $\ldots$ \\
\hline
\end{tabular}

a Positions uncertain by roughly $\pm 3^{\prime \prime}$ ( $\sim 95 \%$ confidence) .

b Magnitudes on system described in Elias et al. 1982 and Frogel, Elias, and Phillips 1982.

' Corrected for reference beam contamination; see text.

${ }^{d}$ Uncertainties in $20 \mu \mathrm{m}$ magnitudes include large standard-star residuals and uncertainties in air-mass corrections. Statistical accuracy of measurements is $>4 \sigma$.

coincides with a visible star of $V \approx 15 \mathrm{mag}$ to better than 0 ".5 (judging from the centering on the $4 \mathrm{~m}$ telescope); this star is assumed to be the same as the infrared source. The visual spectrum of this star is shown in Figure 3. IRAS 05346-6949 has no counterpart on the $R$ or $I$ films of the SRC Southern Survey. In addition, it is so faint at the shortest infrared wavelengths that at 1.2 and $1.6 \mu \mathrm{m}$, the measurements are dominated by flux in a reference beam. Extrapolation to $2.2 \mu \mathrm{m}$ indicates that the measured flux should be increased by $50 \%$ (0.45 mag) to correct for the contamination. The magnitude listed in Table 1 is the corrected magnitude; the uncertainty includes the uncertainty of $\sim 20 \%$ in the correction. No corrections for contamination were made to the data at longer wavelengths, as they should be less than $1 \%$.

\section{DISCUSSION}

Since there are a number of Galactic late $\mathbf{M}$ stars in the direction of the LMC, it is worth presenting the arguments for the two IRAS sources being in fact LMC members.

TABLE 2

Selected IRAS Data

\begin{tabular}{|c|c|c|}
\hline \multirow[b]{2}{*}{ WAVELENGTH $(\mu \mathrm{m})$} & \multicolumn{2}{|c|}{ Flux Densities (Jy) ${ }^{\mathbf{a}}$} \\
\hline & $I R A S 04553-6825$ & IRAS $05346-6949$ \\
\hline ……… & $9.2 \pm 0.3$ & $7.8 \pm 0.2$ \\
\hline $25 \ldots \ldots \ldots+\ldots \ldots$ & $14.4 \pm 0.5$ & $20.8 \pm 0.9$ \\
\hline $60 \ldots \ldots \ldots \ldots \ldots$ & $<12.2^{\mathrm{b}^{-}}$ & $25.6 \pm 6.0$ \\
\hline $100 \ldots \ldots \ldots \ldots \ldots \ldots$ & $<34.2^{\mathrm{b}}$ & $30.6 \pm 5.3$ \\
\hline
\end{tabular}

${ }^{a}$ Flux densities assuming $v F_{v}=$ constant across individual IRAS passbands. See IRAS Explanatory Supplement (chap. VI.C) for discussion of color corrections.

${ }^{b}$ Upper limits set by confusion with nearby sources. Analysis of IRAS Working Survey Data Base suggests $F_{60} \approx 2.5 \mathrm{Jy}$.

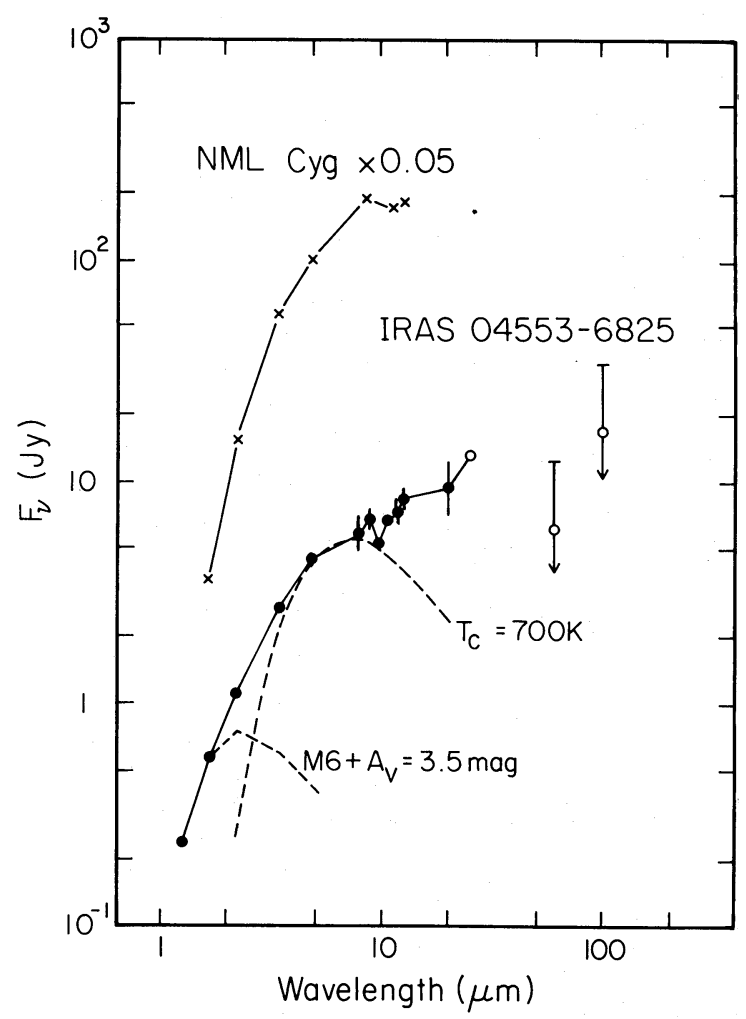

FIG. 1.-Energy distribution of IRAS 04553-6825 (lower curve). The filled circles are the data from Table 1, and the open circles are the 25-100 $\mu \mathrm{m}$ IRAS data summarized in Table 2. The energy distribution from 1.6 to $12 \mu \mathrm{m}$ of NML Cyg (IRC + 40448), a Galactic supergiant $\mathrm{OH}$ maser, is shown for comparison (upper curve); the data are taken from Merrill and Stein $(1976 b)$. The NML Cyg fluxes have been multiplied by 0.05 . Also shown are the distributions of a reddened $\mathrm{M}$ star and a $700 \mathrm{~K}$ blackbody, which together fit the observed energy distribution of IRAS $04553-6825$ well shortward of $10 \mu \mathrm{m}$ (see text).

Hacking et al. (1985) have examined the properties of the IR $A S$ sources above $30^{\circ}$ Galactic latitude which are brighter than $28 \mathrm{Jy}$ at $12 \mu \mathrm{m}$. There are 271 such objects, of which two are galaxies.

Both $04553-6825$ and $05346-6949$ have $25 \mu \mathrm{m}$ to $12 \mu \mathrm{m}$ flux density ratios greater than 1 . In contrast, of the 269 galactic sources listed by Hacking et al., only 16 have 25 to $12 \mu \mathrm{m}$ flux density ratios greater than 0.60 (that is, [12] - [25] colors of $1.0 \mathrm{mag}$ or greater), about $6 \%$ of the total, and none have flux densities at $25 \mu \mathrm{m}$ greater than at $12 \mu \mathrm{m}$ ([12] $-[25]>1.56 \mathrm{mag})$. The density of red, Galactic sources at high latitudes is thus extremely low.

A search through the $I R A S$ catalog for red, high-latitude sources down to lower flux densities than the Hacking et al. limit shows them to still be quite rare. More than $30^{\circ}$ from the galactic plane, there are 67 catalog sources brighter than $3 \mathrm{Jy}$ at $12 \mu \mathrm{m}$ with a 25 to $12 \mu \mathrm{m}$ flux density ratio greater than 0.71 $([12]-[25]>1.2)$; down to $1 \mathrm{Jy}$ at $12 \mu \mathrm{m}$ there are $158 \mathrm{such}$ sources, including Magellanic Cloud sources. Outside of the Magellanic Clouds, the red sources are predominantly cataloged galaxies plus a few planetary nebulae. The density of the remainder is less than $10^{-3} \mathrm{deg}^{-2}$, and none are as red as IRAS $05346-6949$. The radial velocity of IRAS $04553-6825$ (see below) provides additional strong evidence for LMC membership.

All of the other Magellanic Cloud IRAS point sources brighter than $4 \mathrm{Jy}$ at $12 \mu \mathrm{m}$ are significantly bluer or redder 


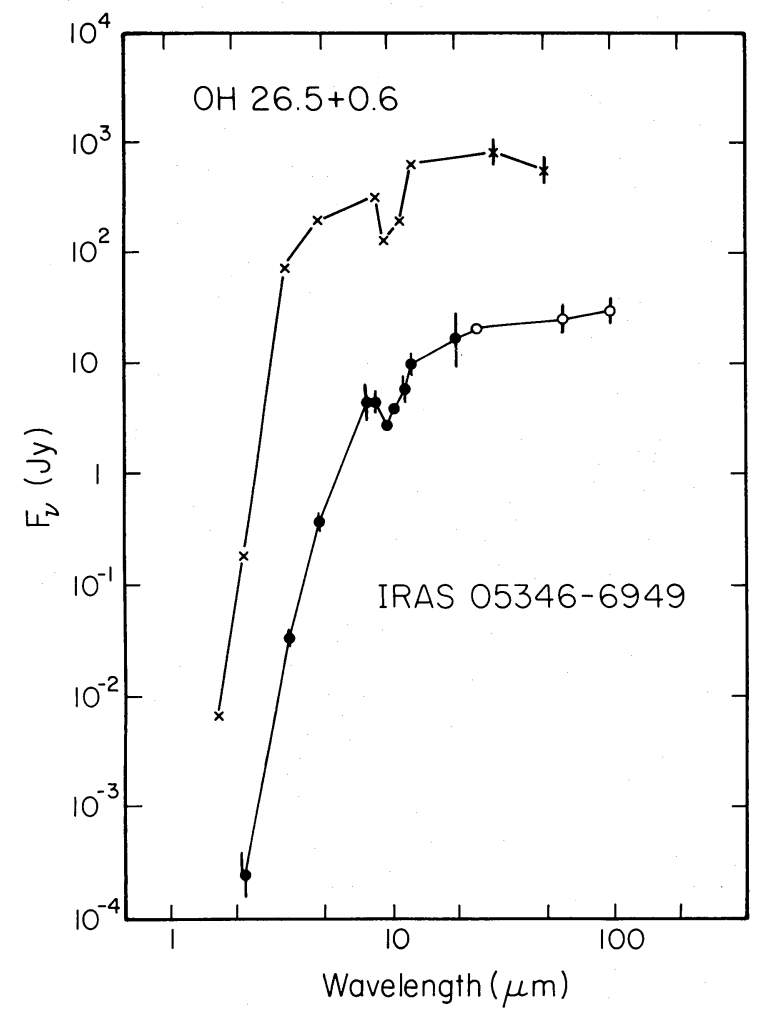

FIG. 2.-Energy distribution of IRAS 05346-6949 (lower curve), as in Fig. 1. An energy distribution for $\mathrm{OH} 26.5-0.6$ from 1.6 to $50 \mu \mathrm{m}$ is shown (upper curve), taken from Werner et al. (1980). OH 26.5-0.6 is much less luminous than IRAS 05346-6949. than these two objects. "Blue" objects are those with $12 \mu \mathrm{m}$ flux densities typically 2-4 times their $25 \mu \mathrm{m}$ flux densities. Most of these have been observed from the ground or have been otherwise convincingly identified as foreground stars. Those objects which are redder can generally be identified with known, visible or radio $H$ II regions. A few (less than a dozen) of the sources fainter than $3 \mathrm{Jy}$ do have colors similar to those of IRAS $04553-6825$ or $05346-6949$. Their exact number is difficult to determine, because of incompleteness due to confusion in crowded LMC regions. The density of these objects is less than $0.1 \mathrm{deg}^{2}$, so it is not surprising that none were detected by previous infrared surveys of portions of the LMC (e.g., Frogel and Richer 1983; McGregor and Hyland 1981). IRAS $05346-6949$ is so faint at wavelengths shortward of 3.5 $\mu \mathrm{m}$ that it could not have been found in any of the groundbased surveys carried out to date.

\section{a) IRAS $04553-6825$}

The spectrum of this object (Fig. 3) shows it to be a late-type $\mathrm{M}$ star, probably around M7.5. Emission lines of $\mathrm{H} \alpha,\left[\mathrm{O}_{\mathrm{I}}\right]$ 6300 , and [ $\mathrm{N} \mathrm{II}] 6548$ and 6584 are prominent. These lines are clearly associated with the star, in that they show no extent along the slit in the original spectrum. Measurements of the radial velocities of the four lines give a redshift of $315 \pm 18 \mathrm{~km}$ $\mathrm{s}^{-1}$ (mean internal error). The true uncertainty in the measurement, which must include the velocity zero-point error, is undoubtedly larger, but unlikely to be more than $50 \mathrm{~km} \mathrm{~s}^{-1}$. The large velocity indicates LMC membership for this object.

The energy distribution of IRAS $04553-6825$ in Figure 1 is much redder than that of a normal M star (e.g., Elias, Frogel, and Humphreys 1985). There is excess emission longward of $1.6 \mu \mathrm{m}$. A simple explanation is that there is emission from a substantial circumstellar shell around the $\mathbf{M}$ star. It is obvious that most of the observed flux is emitted by the shell, which implies that it must have a significant optical depth to radi-

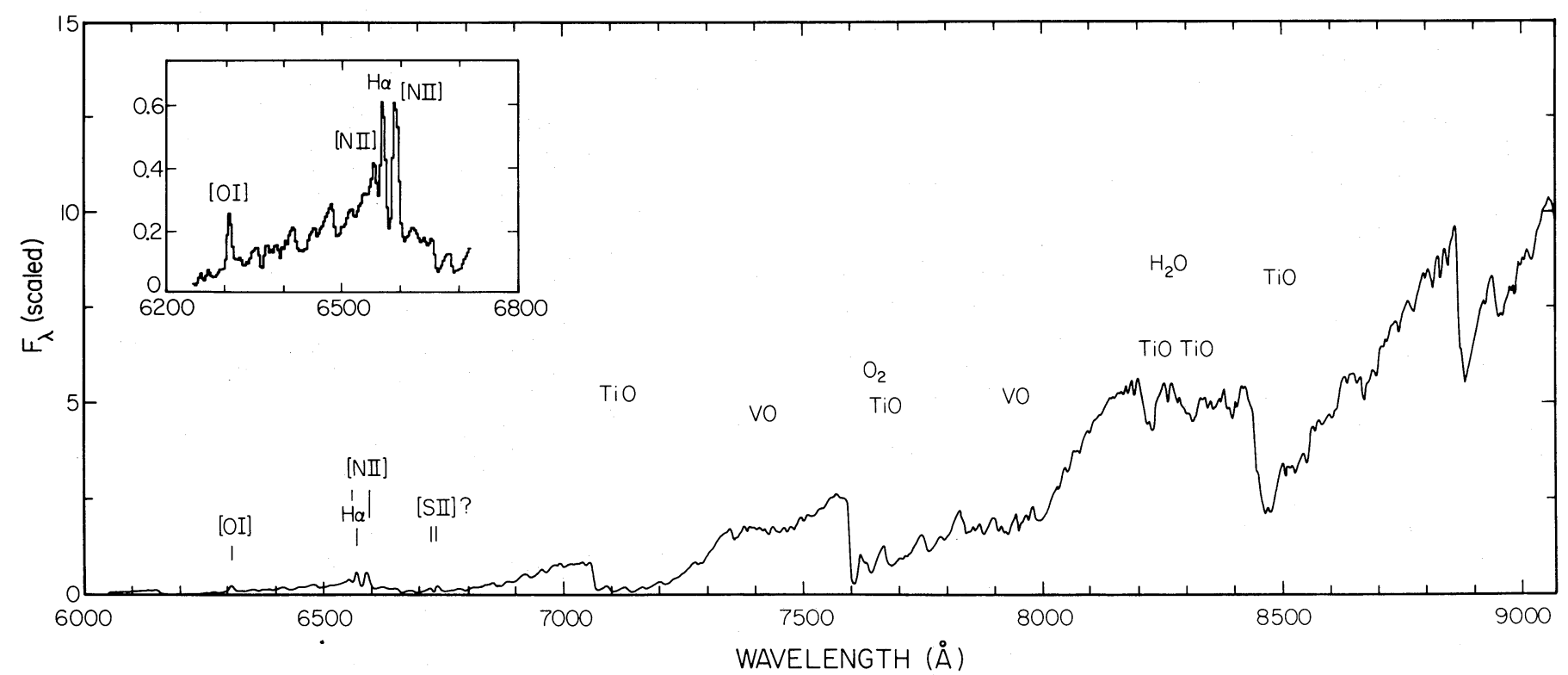

FIG. 3.-Spectrum of IRAS $04553-6825$ from 6000 to $9000 \AA$. The units are flux per unit wavelength interval times an unknown multiplicative constant, which is due to the uncertainty in the overall absolute flux calibration. The four strong emission lines used in determining the radial velocity are shown in the inset on an expanded scale. The spectral:ype of M7.5 is based on a comparison of the strengths of the TiO and VO bands with those seen in other stars of known spectral type observed at the same resolution and signal to noise. Bands of $\mathrm{O}_{2}$ and $\mathrm{H}_{2} \mathrm{O}$ are bands due to Earth's atmosphere, which have not been completely corrected for by the flux calibration procedure. 
ation from the star. A simple model can be constructed by giving the dust a characteristic temperature, and treating the star as an M6 photosphere plus the extinction due to the shell, using colors adopted from Lee (1970) and Elias, Frogel, and Humphreys (1985). A consistent set of values is obtained for a dust color temperature of $700 \mathrm{~K}$ and a visual extinction for the star of roughly $3 \mathrm{mag}$. The dust contributes roughly $30 \%$ of the flux at $K$ and $80 \%$ at $L$. Longward of $10 \mu \mathrm{m}$ there is additional flux, indicating the presence of cooler dust as well. Integration over the emission from the dust shell component shows that it is responsible for $75 \%$ of the observed flux from the system, which means that its flux-averaged optical depth must be roughly 1.5 , consistent with the $3 \mathrm{mag}$ of visual extinction inferred from fitting the $J H K$ colors of the star.

The bolometric magnitude of the object, -9.7 , was derived assuming a distance modulus of +18.6 , and integrating over the combined ground-based and IRAS data. The $V-K$ color was assumed to be $\sim 8$, and the 60 and $100 \mu$ m fluxes were taken as half of the cataloged upper limits. Since most of the flux from this object is emitted between 2 and $25 \mu \mathrm{m}$, different assumptions regarding the visual and far-infrared fluxes affect the bolometric magnitude by less than $0.1 \mathrm{mag}$. The luminosity is significantly greater than that of most late-type variable stars in the Galaxy, and also well above the theoretical and observational limit of the asymptotic giant branch (AGB) near $M_{\text {bol }}$ of -7.1 (e.g., Wood, Bessell, and Fox 1983). In fact, IRAS $04553-6825$ appears to be at least as luminous as any other $M$ star in the LMC (cf. Elias, Frogel, and Humphreys 1985).

The narrow-band $10 \mu \mathrm{m}$ measurements show no evidence for a strong silicate feature, either in absorption or emission. The $8.6 \mu$ m measurement is slightly high (about $1 \sigma$ ) and the 9.6 $\mu$ m measurement is definitely low (roughly $3 \sigma$ ). This suggests that the silicate feature may be self-absorbing in the center (Merrill and Stein 1976a).

The effect of the dust emission on the $\mathrm{H}_{2} \mathrm{O}$ and $\mathrm{CO}$ indices is twofold: first, the addition of a red continuum tends to increase the $\mathrm{H}_{2} \mathrm{O}$ index and weaken the $\mathrm{CO}$ index, and second, the fact that the star is contributing only $70 \%$ of the total flux at 2.2 $\mu \mathrm{m}$ reduces both indices. The tabulated indices do not therefore reflect the true indices of the $\mathrm{M}$ star component. Using the model described above, the stellar $\mathrm{CO}$ index is roughly 0.2 , and the $\mathrm{H}_{2} \mathrm{O}$ index is roughly 0.6 . The $\mathrm{CO}$ index is normal for a late $\mathrm{M}$ star; such strong $\mathrm{H}_{2} \mathrm{O}$ is commonly accompanied by variability.

The IRAS 12 and $25 \mu \mathrm{m}$ data given in the Working Survey Data Base (IRAS Explanatory Supplement, Chap. X.B) show no evidence for significant flux changes between 1983 June and 1983 November; the measured average change at the two wavelengths is a decrease of $5 \% \pm 4 \%$. A comparison with the 1984 December ground-based data is best done by using the 20 $\mu \mathrm{m}$ and narrow-band $10 \mu \mathrm{m}$ observations to synthesize an equivalent $12 \mu \mathrm{m} I R A S$ flux, using the response curve tabulated in IRAS Explanatory Supplement (Chap. II.C). There is an apparent $12 \mu \mathrm{m}$ flux decrease between 1983 November and 1984 December of $15 \% \pm 8 \%$. Since luminous, largeamplitude variables have periods of 500-2000 days (Engels et al. 1983; Wood, Bessell, and Fox 1983; Elias, Frogel, and Humphreys 1985), the three data points cover probable periods well and rule out any variation greater than $50 \%$. Observations at shorter wavelengths usually show large amplitudes for a given star and can also be made to greater precision, so future observations should set more stringent limits on the variability of this object.
The overall energy distribution, the details of the silicate feature observations, and the weakened $\mathrm{CO}$ and $\mathrm{H}_{2} \mathrm{O}$ indices resemble those found in extreme galactic $\mathbf{M}$ stars, such as NML Cyg or IRC + 50137 (Merrill and Stein 1976a). Many of these galactic stars are $\mathrm{OH}$ masers, so a search for emission centered at $\sim 320 \mathrm{~km} \mathrm{~s}^{-1}$ could be-productive. Werner et al. (1980) have shown that the peak OH maser emission from such stars is roughly equal to one-fourth of the $35 \mu \mathrm{m}$ flux. A peak flux of $\sim 2 \mathrm{Jy}$ in the $1612 \mathrm{MHz} \mathrm{OH}$ lines is thus possible. Fluxes at this level are detectable by searches such as those by Caswell and Haynes (1981) and Haynes and Caswell (1981). However, only about one-third of candidate $\mathrm{OH} / \mathrm{IR}$ stars produce measurable $\mathrm{OH}$ maser emission (Herman and Habing 1985).

\section{b) IRAS 05346-6949}

The LMC membership of the object is certain, but its precise classification is not secure because of a lack of data shortward of $5.5 \mu \mathrm{m}$. However, its other characteristics strongly imply that it is also an LMC analog to the Galactic supergiant $\mathrm{OH} / \mathrm{IR}$ stars. Two lines of argument indicate that it is a highly evolved object rather than a young object. First, the energy distribution is significantly different from that of the starforming regions in the LMC and SMC detected by IRAS. These usually have 25 to $12 \mu \mathrm{m}$ flux density ratios greater than 4 -a typical value is 6 - and also have large 60 to $25 \mu \mathrm{m}$ flux density ratios. In contrast, IRAS $05346-6949$ has a 60 to 25 $\mu \mathrm{m}$ flux density ratio of 1.3 and 25 to $12 \mu \mathrm{m}$ flux density ratio less than 3. Also, there are no other indicators of recent star formation activity, such as $\mathrm{H} \alpha$ emission or radio continuum emission, in the vicinity of the IRAS source. Energy distributions like that of IRAS 05346-6949 are seen in Galactic $\mathrm{OH}$ maser sources (e.g., Werner et al. 1980). Following Werner et al., the predicted peak $\mathrm{OH}$ flux should be $\sim 5 \mathrm{Jy}$.

The presence of a silicate feature in absorption (Fig. 2) is an indication that the object is oxygen-rich. This is consistent with the red color between 12 and $25 \mu \mathrm{m}$, since the IRAS Low Resolution Spectrometer did not find any carbon rich objects as red as 05346-6949 (IRAS Explanatory Supplement: Chap. IX.D). The $\sim 1.0 \mathrm{mag}$ depth of the feature agrees with that found for objects of similar $L-M$ color by Jones et al. (1982).

The bolometric magnitude of -9.4 for the object was obtained by integrating over the combined ground-based and $I R A S$ data from $2.2 \mu \mathrm{m}$ out to $100 \mu \mathrm{m}$. Nearly half of the total luminosity is emitted longward of $20 \mu \mathrm{m}$, so the $I R A S$ data are crucial. The $60100 \mu \mathrm{m}$ flux densities are uncertain, because of confusion with other, nearby sources, so the bolometric magnitude is known only to roughly $\pm 0.2 \mathrm{mag}$. The bolometric magnitude is comparable to that of the most luminous galactic OH masers (e.g., Jones, Hyland, and Gatley 1983; Engels et al. 1983; Herman and Habing 1985) and makes the object one of the most luminous evolved stars in the LMC.

For IRAS $05346-6949$, the IRAS 12 and $25 \mu \mathrm{m}$ data show no significant change between 1983 May and 1983 October; the average change at the two wavelengths is a decrease of $3 \% \pm 4 \%$. The 1984 December $10 \mu \mathrm{m}$ data indicate a decline in flux at $12 \mu \mathrm{m}$ of $12 \pm 8 \%$, estimated as for IRAS $04553-6825$. Galactic OH masers typically show amplitudes of over $1 \mathrm{mag}$ at these wavelengths (e.g., Harvey et al. 1974; Engels et al. 1983), which are clearly ruled out. The higher luminosity of this object may thus imply a different type of variability.

Gehrz et al. (1985) found a correlation of the redness of 
galactic $\mathrm{OH}$ masers with distance and suggested that some of the reddening of the apparently cooler, more distant objects is due to interstellar reddening. IRAS 05346-6949 is as red as the reddest galactic $\mathrm{OH}$ sources (cf. Engels et al. 1983; Jones et al. 1983; Gehrz et al. 1985), yet the reddening due to the line-ofsight distance through both the Galaxy and the LMC is small, which indicates that the dust extinction to the central object must be local to it, and thus probably circumstellar. If there is significant extinction outside of the circumstellar shell, the bolometric luminosity estimated above is an underestimate, and the true luminosity becomes still greater.

\section{CONCLUSIONS}

Two infrared sources detected by IRAS in the LMC appear to be extremely luminous, highly evolved stars. Both have extensive dust shells, from which most of the objects' luminosity is emitted. They are the first such objects detected outside the Galaxy. Monitoring for variability and a search for $\mathrm{OH}$ maser activity will be worthwhile.

Both objects have luminosities substantially greater than those seen in the most common type of Galactic $\mathrm{OH}$ masers and equivalent to those of the most luminous Galactic OH/IR stars. They appear to be evolved supergiants rather than lower mass AGB stars.

The two LMC IRAS sources show little variability over 2 yr period and are also at least a magnitude more luminous than the most luminous SMC long-period variable (HV 11417) at maximum. They do not seem to be counterparts (with thicker dust shells) of the extremely luminous SMC long-period vari- ables. This provides further support for the belief that the SMC long-period variables are extreme AGB stars (Elias, Frogel, and Humphreys 1980, 1985). The LMC counterparts of the SMC long-period variables and the lower luminosity Galactic $\mathrm{OH} / \mathrm{IR}$ stars will be a factor of 10 or more fainter at $12 \mu \mathrm{m}$ than the objects discussed here and will thus have been detected by IRAS only in regions of low confusion. There are indeed up to a dozen candidates for such objects among the $I R A S$ sources with $12 \mu \mathrm{m}$ flux densities $<2$ Jy. These LMC $I R A S$ sources should be intermediate in their properties between the SMC long-period variables and typical Galactic $\mathrm{OH} / \mathrm{IR}$ stars.

Since there are only two stars like IRAS $04553-6825$ or IRAS 05346-6949 in the LMC, it is not surprising that there are none in the SMC, which is several times less luminous and less massive. Since the Galaxy is much more luminous than the LMC, it must contain more than the handful of supergiants already known among the galactic $\mathrm{OH} / \mathrm{IR}$ stars; the difficulty is in distinguishing them from the dozens of less luminous stars.

We are grateful to B. T. Soifer and C. Oken for considerable help in understanding $I R A S$ data. The ground-based observations were partially supported by NSF grant AST 83-12699; work with the IRAS data was partially supported by NASA. We also acknowledge the key roles played by B. Atwood and $T$. Ingerson in making the air Schmidt camera and GEC CCD a superb instrument.

\section{REFERENCES}

Caswell, J. L., and Haynes, R. F. 1981, M.N.R.A.S., 174, 33.

Elias, J. H., Frogel, J. A., and Humphreys, R. M. 1980, Ap. J. (Letters), 242, L13.

L1985, Ap. J. Suppl., 57, 91.

Elias, J. H., Frogel, J. A., Matthews, K., and Neugebauer, G. 1982, A.J., 87, 1029.

Engels, D., Kreysa, E., Schiltz, G. V., and Sherwood, W. A. 1983, Astr. Ap., 124, 123

Frogel, J. A., Elias, J. H., and Phillips, M. M. 1982, Ap. J., 260, 70.

Frogel, J. A., and Richer, H. B. 1983, Ap. J., 275, 84.

Gehrz, R. D., Kleinmann, S. G., Mason, S., Hackwell, J. A., and Grasdalen, G. L. 1985, Ap. J., 290, 296.

Hacking, P., et al. 1985, Pub. A.S.P., 97, 616.

Harvey, P. M., Bechis, K. P., Wilson, W. J., and Ball, J. A. 1974, Ap. J. Suppl., 27,331 .

Haynes, R. F., and Caswell, J. L. 1981, M.N.R.A.S., 197, 23.

Herman, J., and Habing, H. J. 1985, Rep. Prog. Phys., in press.

Infrared Astronomical Satellite. (IRAS) Catalogs and Atlases Explanatory Supplement. 1985, ed. C. A. Beichman, G. Neugebauer, H. J. Habing, P. E. Clegg and T. J. Chester (Washington: Government Printing Office), in press.
Jones, T. J., Hyland, A. R., Caswell, J. L., and Gatley, I. 1982, Ap. J., 253, 208.

Jones, T. J., Hyland, A. R., and Gatley, I. 1983, Ap. J., 273, 660.

Lee, T. A. 1970, Ap. J., 162, 217.

McGregor, P. J., and Hyland, A. R. 1981, Ap. J., 250, 116.

Merrill, K. M., and Stein, W. A. $1976 a$, Pub. A.S.P., 88, 285.

. 1976b, Pub. A.S.P., 88, 294.

Neugebauer, G., et al. 1984, Ap. J. (Letters), 278, L1.

Neugebauer, G., and Leighton, R. B., 1969, Two-Micron Sky Survey (NASA SP-3047).

Price, S. D. 1977, The AFGL Four Color Infrared Sky Survey: Supplemental Catalog (AFGL-TR-77-0160).

Price, S. D., and Walker, R. G. 1976, The AFGL Four Color Infrared Sky Survey: Catalog of Observations at 4.2, 11.0, 19.8, and 27.4 $\mu \mathrm{m}$ (AFGL-TR76-0208).

Werner, M. W., Beckwith, S., Gatley, I., Sellgren, K., Berriman, G., and Whiting, D. L. 1980, Ap. J., 239, 540

Wood, P. R., Bessell, M. S., and Fox, M. W. 1983, Ap. J., 277, 99.

J. H. Elias: 320-47 Downs, California Institute of Technology, Pasadena, CA 91125

J. A. Frogel: National Optical Astronomy Observatories, P.O. Box 26732, Tucson, AZ 85726

P. B. W. Schwering: Sterrewacht, Huygens Laboratorium, Wassenaarseweg 78, Leiden 2405, Netherlands 